\title{
Shared and Searchable Encrypted Data for Untrusted Servers ${ }^{\star}$
}

\author{
Changyu Dong, Giovanni Russello, and Naranker Dulay \\ Department of Computing, Imperial College London \\ 180 Queen's Gate, London, SW7 2AZ, UK \\ \{changyu.dong, g.russello,n.dulay\}@imperial.ac.uk
}

\begin{abstract}
Current security mechanisms pose a risk for organisations that outsource their data management to untrusted servers. Encrypting and decrypting sensitive data at the client side is the normal approach in this situation but has high communication and computation overheads if only a subset of the data is required, for example, selecting records in a database table based on a keyword search. New cryptographic schemes have been proposed that support encrypted queries over encrypted data but all depend on a single set of secret keys, which implies single user access or sharing keys among multiple users, with key revocation requiring costly data re-encryption. In this paper, we propose an encryption scheme where each authorised user in the system has his own keys to encrypt and decrypt data. The scheme supports keyword search which enables the server to return only the encrypted data that satisfies an encrypted query without decrypting it. We provide two constructions of the scheme giving formal proofs of their security. We also report on the results of a prototype implementation.
\end{abstract}

\section{Introduction}

Data growth is inevitable for nearly all organisations. According to Forrester Research, enterprise storage needs grow at 52 percent per year [1]. To reduce the increasing costs of storage management, many organisations choose to outsource their data storage to third party service providers. Recent research from TheInfoPro shows that nearly $20 \%$ of Fortune 1000 organisations outsource at least some portion of their storage management activities [2].

One of the biggest challenges raised by data storage outsourcing is security and trust. Business data is a valuable asset for many companies. While companies may trust a Storage Service Provider's (SSP) reliability, availability, faulttolerance and performance, they cannot trust that an SSP is not going to use the data for other purposes, especially when the value of the data is high. Traditional access controls which are used to provide confidentiality are mostly designed for in-house services and depend greatly on the system itself to enforce authorisation

\footnotetext{
* This research was supported by the UK's EPSRC research grant EP/C537181/1. The authors would like to thank the members of the Policy Research Group at Imperial College for their support.
} 
policies, effectively relying on a trusted infrastructure. In the absence of trust, traditional security models are no longer valid. Another common approach to provide data confidentiality is cryptography. Server side encryption is not appropriate when the server is not trusted. The client must encrypt the data before sending it to the SSP and later the encrypted data can be retrieved and decrypted by the client. This could ease a company's concern about data leakage, but introduces a new problem. Because the encrypted data is not meaningful to the servers, many useful data management functionalities are not possible. For example, if a client wants to retrieve documents or records containing certain keywords, how can this request be processed? Can we keep the data incomprehensible to servers and their administrators while efficiently retrieving the data? Consider the following scenarios:

Scenario 1. Company $A$ is considering outsourcing its data processing centre to a service provider B. This will cut its annual IT cost by up to 25\%. But the CIO is concerned about data security. The company's databases contain valuable production data and customer information. It would be unacceptable if competitors got hold of the data. Administrative controls such as formal contracts, confidential agreements and continuous auditing provide a certain level of assurance, but the CIO would also like to encrypt the sensitive data and have fast searches over it.

Scenario 2. Bob subscribes to a Personal Health Record service from company C. The service allows Bob to maintain his electronic medical records and share them with his doctors through a web interface. Bob wants to encrypt his records, ensuring the staff of company $C$ will not be able to know what is inside.

A trivial solution is to download all the data to the client's computer and decrypt it locally. This does not scale to large datasets. Recently, several innovative schemes have been proposed to address the above problems. The basic idea is to divide the cryptographic component between the client and server. The client performs the data encryption/decryption and manages the keys. The server processes search queries by carrying out some computation on the encrypted data. The server knows nothing about the keys or the plaintexts of the data nor the queries, but is still able to return the correct results.

These schemes also have an important limitation. The operations, e.g. encryption, decryption and query generation, more or less rely on some secret keys. This implies that the operations can only be executed by one user, or by a group of users who share the secret keys somehow. A single user is usually not an adequate assumption for data outsourcing. Perhaps the biggest problem for supporting multiple user access to encrypted data is key management. Sharing keys is generally not a good idea since it increases the risk of key exposure. In response to this, keys must be changed regularly. The keys must also be changed if a user is no longer qualified to access the data. However, changing keys may result in decrypting all the data and re-encrypting it using the new keys. For large data sets, this is not practical. 
In this paper, we propose a scheme for multi-user searchable data encryption. Our scheme does not require a fully trusted server. The server can search an encrypted keyword on the encrypted data. More importantly each authorised user in the system has his own unique keys which simplifies key revocation and avoids data re-encryption. All the authorised users can insert encrypted data, decrypt the data inserted by other users and search encrypted data without knowing the other users' keys. The keys of one user can easily be revoked without affecting other users or the encrypted data at the server.

\section{Related Work}

Song et al. 3] introduced the first practical scheme for searching on encrypted data. The scheme enables clients to perform searches on encrypted text without disclosing any information about the plaintext to the untrusted server. The untrusted server cannot learn the plaintext given only the ciphertext, it cannot search without the user's authorisation, and it learns nothing more than the encrypted search results. The basic idea is to generate a keyed hash for the keywords and store this information inside the ciphertext. The server can search the keywords by recalculating and matching the hash value. Yang et al. 4] proposed an elegant scheme for performing queries on encrypted data and also provided a secure index to speed up queries by two-step mapping. Goh's scheme [5] enables searches on encrypted data by constructing secure indexes based on bloom filter.

In the bucketization approach for searching encrypted databases 6 7/89], an attribute domain is partitioned into a set of buckets each of which is identified by a tag. These bucket tags are maintained as an index and are utilised by the server to process the queries. Bucketization has relatively small performance overhead and enables more complex queries such as range queries and comparison queries at the cost of revealing more information about the encrypted data.

All the schemes above rely on secret keys however, which implies single user access or sharing keys among a group of users. Boneh et al. [10] presented a scheme for searches on encrypted data using a public key system that allows mail gateways to handle email based on whether certain keywords exist in the encrypted message. The application scenario is similar to [3], but the scheme uses identity-based encryption instead of symmetric ciphers. Using asymmetric keys allows multiple users to encrypt data using the public key, but only the user who has the private key can search and decrypt the data. Curtmola et al. 11] partly solved the multi-user problem by using broadcast encryption. The set of authorised users share a secret key $r$ (which is used in conjunction with a trapdoor function). Only people who know $r$ will be able to access/query the data. A user can be revoked by changing $r$, and using broadcast encryption to send the new key $r^{\prime}$ to the set of authorised users. The revoked user does not know $r^{\prime}$, and hence cannot search. In this scheme, the database is searchable, but is read-only and cannot be updated. In our scheme, any authorised user can read, search and update the database. 


\section{Multi-user Searchable Data Encryption Scheme: Basic Construction}

In this section, we introduce the basic construction of the multi-user searchable data encryption scheme which is built upon proxy encryption. The scheme does not require sharing keys among the users. We also formalise the notions of security and provide proofs later in this section.

\subsection{An RSA-Based Proxy Encryption Scheme}

The notion of proxy encryption was first introduced in [12]. In a proxy encryption scheme, a ciphertext encrypted by one key can be transformed by a proxy function into the corresponding ciphertext for another key without revealing any information about the keys and the plaintext. Proxy encryption schemes can be built on top of different cryptosystems such as El Gamal [13] and RSA [14. Applications of proxy encryption include: secure email lists [15, access control systems [16] and attribute based publishing of data [17]. A comprehensive study on proxy cryptography can be found in [18].

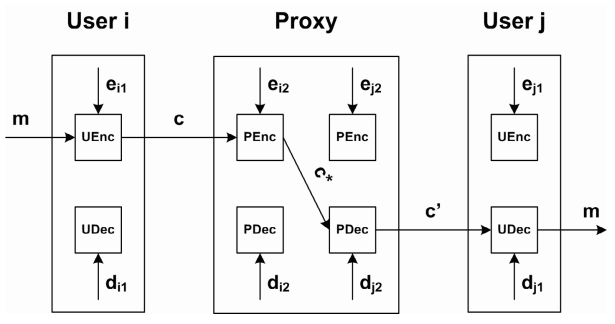

Fig. 1. Encryption/Decryption in Our RSA-based Proxy Encryption Scheme

Our scheme uses an RSA-based proxy encryption scheme. Let's use $\mathcal{E}=($ IGen, $U G e n, U E n c, U D e c, P E n c, P D e c)$ to denote the proxy encryption scheme. Fig. 1] shows the encryption/decryption process in the proxy encryption scheme.

- IGen is the master key generation algorithm which is identical to the key generation algorithm in the standard RSA. It takes a security parameter $k$ and generates $(p, q, n, \phi(n), e, d)$. IGen needs only to be run once at the beginning of the system setup. All the outputs except $n$ must be kept secret. In the rest of the paper, we assume all arithmetic to be $\bmod n$ unless stated otherwise.

- UGen is the algorithm for generating the key pairs for the users and the proxy. For each user $i, U G e n$ takes the output of $I G e n$ and finds $e_{i 1}, e_{i 2}, d_{i 1}$, $d_{i 2}$ such that $e_{i 1} e_{i 2} \equiv e \bmod \phi(n)$ and $d_{i 1} d_{i 2} \equiv d \bmod \phi(n)$. This can be efficiently done. Take the $e_{i 1}, e_{i 2}$ pair for example, we can pick $e_{i 1}<\phi(n)$ randomly, where $e_{i 1}$ is relatively prime to $\phi(n)$, i.e. $\operatorname{gcd}\left(e_{i 1}, \phi(n)\right)=1$. Since 
$e_{i 1} x \equiv 1 \bmod \phi(n)$ always has a solution, then $e_{i 2} \equiv \operatorname{ex} \bmod \phi(n)$ always satisfies $e_{i 1} e_{i 2} \equiv e \bmod \phi(n)$. Note that knowing only $a$ is not sufficient for solving the two variable equation $a x \equiv y \bmod n$. Therefore by knowing only $e_{i 1}$ or $e_{i 2}$, one cannot compute its counterpart ( $e_{i 2}$ or $e_{i 1}$ respectively) and $e$. The user's key pair is $\left(K_{u e i}, K_{u d i}\right)=\left(e_{i 1}, d_{i 1}\right)$. The proxy's corresponding key pair for the user $i$ is $\left(K_{p e i}, K_{p d i}\right)=\left(e_{i 2}, d_{i 2}\right)$. The lower bound of the number of valid key pairs is $\phi(\phi(n))>\sqrt{\phi(n)}$.

- UEnc is the algorithm for user encryption. For a message $m$, user $i$ encrypts it using his encryption key $K_{u e i}=e_{i 1}$. The resulting ciphertext is $c=m^{e_{i 1}}$.

- PEnc is the algorithm for proxy encryption. When the proxy receives a ciphertext $c$ from user $i$, it re-encrypts it using the corresponding encryption key $K_{p e i}=e_{i 2}$ as $c^{*}=c^{e_{i 2}}$.

- PDnc is the algorithm for proxy decryption. Before sending the ciphertext to user $j$, the proxy decrypts it using the corresponding decryption key $K_{p d j}=d_{j 2}$ as $c^{\prime}=\left(c^{*}\right)^{d_{j 2}}$.

- UDec is the algorithm for user decryption. When a user $j$ receives a ciphertext $c^{\prime}$ from the proxy, he decrypts it using his decryption key $K_{u d j}=d_{j 1}$. He will be able to recover the plaintext $m=\left(c^{\prime}\right)^{d_{j 1}}$.

Note that in the system, for any user $i$ and any user $j, e_{i 1} e_{i 2} \equiv e_{j 1} e_{j 2} \equiv$ $e \bmod \phi(n)$ and $d_{i 1} d_{i 2} \equiv d_{j 1} d_{j 2} \equiv d \bmod \phi(n)$. Therefore $c^{*}=c^{e_{i 2}}=m^{e_{i 1} e_{i 2}}=$ $m^{e}, c^{\prime}=\left(c^{*}\right)^{d_{j 2}}=m^{e d_{j 2}}$ and the user $j$ can correctly decrypt $c^{\prime}$ because $\left(c^{\prime}\right)^{d_{j 1}}=$ $m^{e d_{j 2} d_{j 1}}=m^{e d}=m$.

In our system, we use a trusted key management server (KMS) controlled by the data owner to manage the keys. First, the KMS runs IGen to generate a master key pair $(e, d)$ and publishes the only public parameter $n$. When a new user is enrolled into the system, the KMS runs UGen to generate a unique tuple $\left(\left(e_{i 1}, d_{i 1}\right),\left(e_{i 2}, d_{i 2}\right)\right)$ and sends $\left(e_{i 1}, d_{i 1}\right)$ to the user and $\left(e_{i 2}, d_{i 2}\right)$ to the data server through secure channels. If the user is removed from the system at a later stage, the KMS can send a instruction to the data server to remove the key pair $\left(e_{i 2}, d_{i 2}\right)$ at the server side. We will see in the following sections, without the server side key pairs, the user cannot search and decrypt the data.

Although requiring a trusted KMS seems at odds with using an untrusted data storage service, we can argue that the KMS requires less resources and less management effort. Securing the KMS is much easier since a very limited amount of data needs to be protected and the KMS can be kept offline most of time.

\subsection{Data Encryption}

In our system, each data item $D_{x}$ is associated with a set of searching keywords $\left\{W_{1}, W_{2}, \ldots, W_{n}\right\}_{x}$. The encryption algorithm is shown in Fig. 2, The data item could be a document, an email, or a data cell in a database etc..

The data encryption is done at the client side using a semantically secure [19] symmetric encryption algorithm $E$. For each data item $D_{x}$, the user $i$ picks a key $K_{x}$ uniformly randomly from the key space of $E$ and encrypts $D_{x}$ under the 


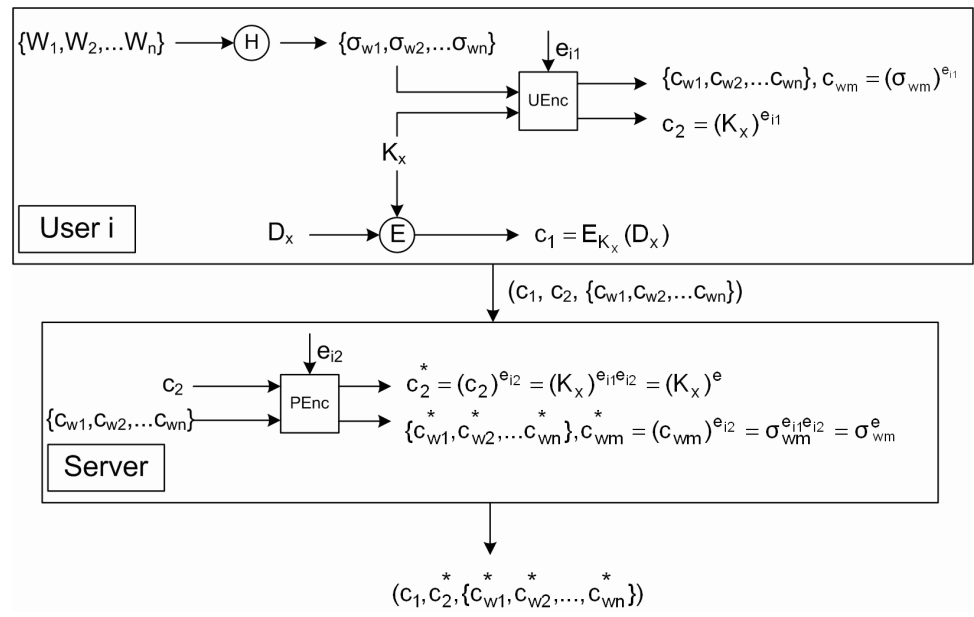

Fig. 2. Basic Data Encryption Scheme

key which generates a ciphertext $c_{1}=E_{K_{x}}\left(D_{x}\right) . K_{x}$ is then encrypted by the user's piece of RSA encryption key as $c_{2}=\left(K_{x}\right)^{e_{i 1}}$.

For each search keyword $W_{m}$, the client uses a hash function $H$ to compute $\sigma_{w m}=H\left(W_{m}\right)$ and computes $c_{w m}=\left(\sigma_{w m}\right)^{e_{i 1}}$. The client then sends the tuple $\left(c_{1}, c_{2},\left\{c_{w 1}, c_{w 2}, \ldots, c_{w n}\right\}\right)$ to the server.

After receiving the tuple, the server first computes $c_{2}^{*}=c_{2}^{e_{i 2}}$. For each encrypted keyword $c_{w m}$, the server computes $c_{w m}^{*}=c_{w m}^{e_{i 2}}$. The final cipher stored on the server is a tuple $\left(c_{1}, c_{2}^{*},\left\{c_{w 1}^{*}, c_{w 2}^{*}, \ldots c_{w m}^{*}\right\}\right)$.

\subsection{Keyword Search}

A user $j$ may want to retrieve all the documents on the server which contain a keyword $W$. To do so, $j$ first computes the hash value of the keyword $\sigma=H(W)$. Then $j$ encrypts $\sigma$ as $Q=\sigma^{e_{j 1}}$ and sends $Q$ to the server.

The server re-encrypts $Q$ as $Q^{*}=Q^{e_{j 2}}$. Then it tests each ciphertext: in the encrypted keywords set $\left\{c_{w 1}^{*}, \ldots, c_{w m}^{*}\right\}_{x}$, if there exists a $c_{w m}^{*}$ such that $c_{w m}^{*}=Q^{*}$, then adds this ciphertext into the result set.

Recall that $e_{i 1} e_{i 2} \equiv e_{j 1} e_{j 2} \equiv e \bmod \phi(n)$, so $Q^{*}=(H(W))^{e}$ and $c_{w m}^{*}=$ $\left(H\left(W_{m}\right)\right)^{e}$ are equal if and only if $W=W_{m}$. If the server cannot find the corresponding key for the user $j$, it cannot correctly compute the searching keyword. Therefore an unauthorised user cannot perform searching on the data.

\subsection{Data Decryption}

If an authorised user $j$ wants to retrieve $D_{x}$, the server gets the tuple $\left(c_{1}, c_{2}^{*},\left\{c_{w 1}^{*}\right.\right.$, $\left.\left.c_{w 2}^{*}, \ldots c_{w m}^{*}\right\}\right)$ from the data storage, computes $c_{2}^{\prime}=\left(c_{2}^{*}\right)^{d_{j 2}}$ and sends $c_{1}, c_{2}^{\prime}$ to $j$. The user $j$ then computes $\left(c_{2}^{\prime}\right)^{d_{j 1}}=\left(c_{2}^{*}\right)^{d}=\left(K_{x}\right)^{e d}=K_{x}$ and can decrypt the data item $D_{x}=E_{K_{x}}^{-1}\left(c_{1}\right)$. An unauthorised user cannot decrypt the data because the server does not have the corresponding proxy decryption key. 


\subsection{Attack Model}

We focus the scope of our scheme on protecting data confidentiality, therefore we will not consider attacks on data integrity and availability which can be handled by other mechanisms. For the scheme, we assume that the KMS and the authorised users are fully trusted. We also assume they can properly protect their secrets, for example, the key pairs and the parameters for generating keys. The server is modelled as "honest-but-curious", i.e. we trust it to correctly execute the instructions from the clients, but do not want it to access the plain data. An adversary $A d v$ is an attacker (or a software agent) that gains privileged access to the data storage: either an outsider or a untrustworthy employee in the data centre. The adversary can also intercept the communications between clients and the server, but it is computationally bounded. In addition, the adversary is restricted to only perform passive attacks, i.e. attacks are based upon observed data. This restriction is reasonable because: (1) in most cases $A d v$ is physically isolated from the users; (2) most communications between the clients and the server are one-round and initialised by the client, i.e. query-reply. The goal of the adversary is to gather direct or indirect information about the stored data.

\subsection{Security Analysis}

We now give the formal notions of security and proof of security for our system. Note that in the basic construction, the ciphertexts are encrypted by two different schemes. In such situations, the security of the whole system depends on the individual scheme. We assume that the symmetric key scheme is semantically secure, and will prove our proxy encryption scheme is One-Way secure.

Readers who are familiar with RSA may have concerns because there are several known attacks on RSA, e.g. common modulus attack 2021, which enables an attacker to recover the plaintext. Because our proxy encryption scheme is RSA-based, readers may be curious about how secure it is. We will prove in lemma 1 that if an attacker can recover a plaintext encrypted under our proxy encryption scheme, then he can recover any message encrypted by any arbitrary RSA key by knowing only the ciphertext and the modulus $n$. This contradicts the RSA assumption, therefore our scheme should be secure against all such attacks.

Definition 1. Let $\mathcal{E}=(I G e n, U G e n, U E n c, U D e c, P E n c, P D e c)$ be the proxy encryption scheme. $\mathcal{E}$ is said to be One-Way secure against any PPT attacker $\mathcal{A}$ if $\operatorname{Succ}_{\mathcal{A}, \mathcal{E}}$ is negligible. Succ ${ }_{\mathcal{A}, \mathcal{E}}$ is defined as follows:

$$
\operatorname{Succ}_{\mathcal{A}, \mathcal{E}}=\operatorname{Pr}\left[m^{\prime}=m \mid \begin{array}{c}
(p, q, n, \phi(n), e, d) \leftarrow \operatorname{IGen}\left(1^{k}\right), \\
\left(\mathcal{K}_{u}, \mathcal{K}_{p}\right) \leftarrow \operatorname{UGen}(\phi(n), e, d), \\
m^{\prime} \leftarrow \mathcal{A}\left(\mathcal{K}_{p}, n, m^{\varepsilon}\right), \varepsilon \in \mathcal{K}_{u}
\end{array}\right]
$$

Loosely speaking, the proxy encryption scheme is one-way secure if by knowing the public parameter $n$, all the key pairs on the server side, ciphertexts encrypted under an authorised user's encryption key and any information can be derived from above, e.g. intermediate ciphertexts calculated using the server side keys, 
but without knowing any key pairs in the authorised user key pair set $\mathcal{K}_{u}$, no PPT adversary can find the corresponding plaintext.

Lemma 1. Under the RSA assumption, the proxy encryption scheme is OneWay secure against Adv.

Proof. We will show that if $A d v$ can break the proxy encryption scheme, i.e. $\operatorname{Succ}_{\mathcal{A}, \mathcal{E}}$ is not negligible, then there is an attacker $\mathcal{B}$ who can solve the RSA problem with non-negligible probability.

Given an RSA ciphertext $c=m^{e}$ where the corresponding key pair is $(e, d)$, the goal of $\mathcal{B}$ is to decrypt it, i.e. to find $m$. $\mathcal{B}$ can pick $x$ pairs of random primes $\frac{n}{2}<\left(e_{\mathcal{B}}, d_{\mathcal{B}}\right)_{i}<n-2^{161}$. The primes are relatively prime to $\phi(n)$ because $\frac{\phi(n)}{2}<\left(e_{\mathcal{B}}, d_{\mathcal{B}}\right)_{i}<\phi(n) . \mathcal{B}$ then sends $c, n,\left(e_{\mathcal{B}}, d_{\mathcal{B}}\right)_{i}, i=1, \ldots, x$ to $A d v$.

$A d v$ can computes $c_{1}=c^{e_{\mathcal{B} 1}}, c_{2}=c_{1}^{d_{\mathcal{B} 1}}$. Next we will show that $c, c_{1}, c_{2}, n$, $\left(e_{\mathcal{B}}, d_{\mathcal{B}}\right)_{i}, i=1, \ldots, x$ can correctly simulate $a d v$ 's knowledge in the proxy encryption scheme. First we will show that $c, c_{1}, c_{2}$ are valid ciphertexts for the proxy encryption scheme. The ciphertexts are valid if there exists a $d^{\prime}$ such that $e_{2}^{d^{\prime}}=m$, i.e. $e e_{\mathcal{B} 1} d_{\mathcal{B} 1} d^{\prime} \equiv 1 \bmod \phi(n)$. Because $e_{\mathcal{B} 1}, d_{\mathcal{B} 1}$ are relatively prime to $\phi(n)$, we can always find $y$ such that $e_{\mathcal{B} 1} d_{\mathcal{B} 1} y \equiv 1 \bmod \phi(n)$. Therefore there always exists $d^{\prime} \equiv d y \bmod \phi(n)$ such that $e e_{\mathcal{B} 1} d_{\mathcal{B} 1} d^{\prime} \equiv e e_{\mathcal{B} 1} d_{\mathcal{B} 1} d y \equiv(e d)\left(e_{\mathcal{B} 1} d_{\mathcal{B} 1} y\right) \equiv$ $1 \bmod \phi(n)$. We also need to show that $\left(e_{\mathcal{B}}, d_{\mathcal{B}}\right)_{i}, i=1, \ldots, x$ are valid server side key pairs, this can be easily proved using the similar method as above therefore is omitted.

Now with the message from $\mathcal{B}, A d v$ can find $m$ with probability $\operatorname{Succ}_{\mathcal{A}, \mathcal{E}}$ and returns the result to $\mathcal{B}$. This means $\mathcal{B}$ can solve the RSA problem with non-negligible probability $S u c c_{\mathcal{A}, \mathcal{E}}$, which contradicts the RSA assumption.

Theorem 1. The basic construction is One-Way secure against Adv.

This is quite straightforward. The ciphertext is encrypted disjointedly by two encryption schemes. The symmetric encryption scheme is semantically secure, i.e. ciphertext indistinguishable, which implies it is One-Way secure against $A d v$. Since the proxy encryption scheme has been proved to be One-Way secure, overall, the basic construction is One-Way secure against $A d v$.

One-Way secure is sufficient to protect a data item, since an adversary cannot recover the symmetric key and then decrypt the data item. But it does leak some information about the keywords. Because the proxy encryption is deterministic, the ciphertexts of keywords are not indistinguishable. All the occurrences of the same keyword generate the same ciphertext. The adversary can make inferences from the keyword distribution by observing the encrypted data service.

In the following section, we will show a enhanced scheme which is semantically secure and makes the above attack impossible.

\section{Enhanced Construction}

The problem with the basic construction comes from the fact that the keyword encryption is not semantically secure. Using some probabilistic padding schemes 
can solve the problem, but then the encrypted keywords are no longer searchable. In this section, we will show a enhanced construction with a new keyword encryption scheme which is both semantically secure and searchable.

\subsection{Keyword Encryption Scheme}

In the new construction, to avoid the problem discussed in section 3.6 , the keywords are no longer encrypted under the proxy encryption scheme. Instead, we encrypt each keyword as a non-interactive zero-knowledge proof style witness. An additional key pair is generated for encrypting the keywords and in the queries. The new keyword encryption scheme is based on Discrete Logarithms.

Let $\mathcal{E}^{\prime}=\left(I G e n^{\prime}, U G e n^{\prime}, U E n c^{\prime}, P E n c^{\prime}\right)$ denote the keyword encryption scheme.

- IGen' is the algorithm for generating the public parameters and the master key. It takes a security parameter $k$ and generates $\left\{p^{\prime}, q^{\prime}, g, x, h, a, g^{a} h^{a}\right\}$. $p^{\prime}$ and $q^{\prime}$ are two large prime numbers such that $q^{\prime}$ divides $p^{\prime}-1 . g$ is a generator of $G_{q^{\prime}}$, the unique order- $q^{\prime}$ subgroup of $Z_{p^{\prime}}^{*} . h \equiv g^{x} \bmod p^{\prime}$ where $x$ is chosen uniformly randomly from $Z_{q^{\prime}}$. $a$ is also a random number from $z_{q^{\prime}} \cdot p^{\prime}, q^{\prime}, g, h, g^{a} h^{a}$ are publicised and $x, a$ must be kept secret. The reason why we publish $g^{a} h^{a}$ instead of $g^{a}$ is that if $g^{a}$ is available to the adversary, then it can generate search queries of any chosen keywords.

- UGen' is the algorithm for generating the key pairs for the users and the proxy. For a user $i$, it finds $a_{i 1} a_{i 2} \equiv a \bmod q^{\prime}$. The user's keyword encryption key is $a_{i 1}$, and the proxy's share is $a_{i 2}$. The number of key pairs is at least $\phi\left(q^{\prime}\right)=q^{\prime}-1$.

- UEnc' is the client-side encryption algorithm.

- PEnc' is the server-side encryption algorithm.

Note that there is no decryption algorithm for this keyword encryption scheme. This is because the ciphertexts of the keywords are only used for testing whether there is a match and do not need to be decrypted.

\subsection{Data Encryption/Decryption}

The new encryption scheme is shown in Fig. 3. The data item encryption/ decryption is the same as in the basic construction. Although plain RSA can sufficiently protect the symmetric keys used to encrypt the data items, it cannot make the ciphertexts indistinguishable and may leak some information. If the adversary can somehow distinguish the encrypted keys and uses the keys as tags, he can distinguish the data items. To prevent such attacks, we pad the keys with OAEP (Optimal Asymmetric Encryption Padding) [22, a probabilistic padding scheme, before encryption. RSA-OAEP has been proved to be indistinguishable under adaptive chosen ciphertext attack in the random oracle model [23. Now the symmetric key is encrypted by the user's piece of the RSA encryption key 


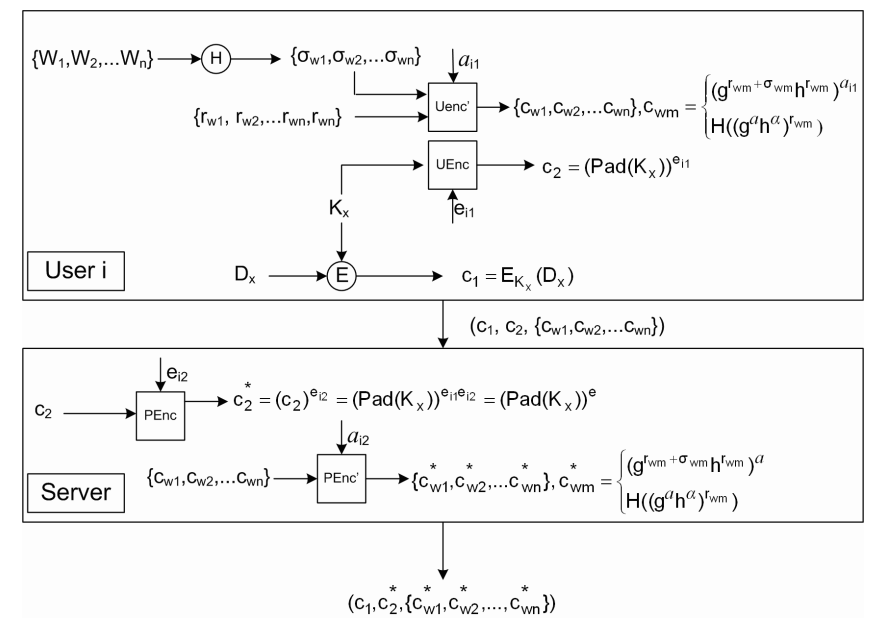

Fig. 3. Data Encryption Algorithm 2

as $c_{2}=\left(\operatorname{Pad}\left(K_{x}\right)\right)^{e_{i 1}}$. The server side proxy encryption/decryption algorithms remain the same, i.e. modular exponentiation.

The keywords are now processed as follows: for each keyword $W_{m}$, the user $i$ computes $\sigma_{w m}=H\left(W_{m}\right)$ using a hash function $H$. The user also picks a random number $r_{w m} \in Z_{q^{\prime}}$ and computes $c_{w m, 1}=\left(g^{r_{w m}+\sigma_{w m}} h^{r_{w m}}\right)^{a_{i 1}} \bmod p^{\prime}$, $c_{w m, 2}=H\left(\left(g^{a} h^{a}\right)^{r_{w m}}\right)$, where $g, h, g^{a} h^{a}, p^{\prime}$ are public parameters in the system and $a_{i 1}$ is the user's keyword encryption key. The user then sends the tuple $\left(c_{1}, c_{2},\left\{c_{w 1}, c_{w 2}, \ldots, c_{w n}\right\}\right)$ to the server, where $c_{w m}$ is the tuple $\left(c_{w m, 1}, c_{w m, 2}\right)$.

The server re-encrypts the data encryption key as in the basic construction. Then it processes the keywords information. For each $c_{w m}$, the server computes $c_{w m, 1}^{*}=c_{w m 1}^{a_{i 2}}=\left(g^{r_{w m}+\sigma_{w m}} h^{r_{w m}}\right)^{a_{i 1} a_{i 2}}=\left(g^{r_{w m}+\sigma_{w m}} h^{r_{w m}}\right)^{a} \bmod p^{\prime}, c_{w m, 2}^{*}=$ $c_{w m, 2}$. The final cipher stored is a tuple $\left(c_{1}, c_{2}^{*},\left\{c_{w 1}^{*}, c_{w 2}^{*}, \ldots c_{w m}^{*}\right\}\right)$, where $c_{w m}^{*}=$ $\left(c_{w m, 1}^{*}, c_{w m, 2}^{*}\right)$.

\subsection{Keyword Search}

To search for a keyword $W$, the user $j$ computes $\sigma=H(W)$. The user then computes the encrypted query $Q=g^{(-\sigma) a_{j 1}} \bmod p^{\prime}$ and sends it to the server. The server computes $Q^{\prime}=Q^{a_{j 2}} \bmod p^{\prime}=g^{(-\sigma) a} \bmod p^{\prime}$. For each $c_{w m}^{*}$, the server computes:

$$
\begin{aligned}
& y_{1}=c_{w m, 1}^{*} Q^{\prime}=\left(g^{r_{w m}+\sigma_{w m}} h^{r_{w m}}\right)^{a} g^{(-\sigma) a}=\left(g^{a r_{w m}+a \sigma_{w m}} h^{a r_{w m}}\right) g^{(-a \sigma)} \bmod p^{\prime} \\
& y_{2}=H\left(\left(y_{1}\right)\right)
\end{aligned}
$$

We can see that if $a \sigma_{w m}-a \sigma=0$, i.e., $W_{m}=W$, then $y_{1}=\left(g^{a r_{w m}} h^{a r_{w m}}\right)=$ $\left(g^{a} h^{a}\right)^{r_{w m}} \bmod p^{\prime}$ and therefore $y_{2}=H\left(\left(g^{a} h^{a}\right)^{r_{w m}}\right)=c_{w m, 2}^{*}$. Then by comparing $y_{2}$ and $c_{w m, 2}^{*}$, the server can decide whether the keyword matches the query. 


\subsection{Security Analysis}

We first prove that the keyword encryption is semantically secure. Semantic security means that the ciphertexts are indistinguishable to the adversary, therefore the adversary learns nothing by looking at the ciphertext.

Lemma 2. Let the keyword encryption $\mathcal{K} \mathcal{E}=\left(\right.$ Pub_para, Sec_para, $\mathcal{K}_{u}, \mathcal{K}_{p}$, Enc) where Pub_para is the public parameter set, Sec_para is the secret parameter set, $\mathcal{K}_{u}, \mathcal{K}_{p}$ are the user and proxy key sets respectively, Enc, Dec are the encryption/decryption algorithms. It is semantically secure against any PPT attacker (i.e. $\operatorname{Succ}_{\mathcal{A}, \mathcal{K} \mathcal{E}}$ is negligible) where

$$
\operatorname{Succ}_{\mathcal{A}, \mathcal{K} \mathcal{E}}=\operatorname{Pr}\left[b^{\prime}=b \mid \begin{array}{c}
m_{0}, m_{1} \in\{0,1\}^{l}, \\
b \stackrel{R}{\leftarrow}\{0,1\}, \\
b^{\prime} \leftarrow \mathcal{A}\left(\text { Pub_para, } \mathcal{K}_{p}, \operatorname{Enc}_{k}\left(m_{b}\right)\right), k \in \mathcal{K}_{u}
\end{array}\right]-\frac{1}{2}
$$

Proof. The ciphertext of a keyword $m_{b}$ in the form of $c_{m_{b}}=\left(\left(g^{r_{m_{b}}+\sigma_{m_{b}}} h^{r_{m_{b}}}\right)^{a_{i 1}}\right.$, $\left.H\left(\left(g^{a} h^{a}\right)^{r_{m_{b}}}\right)\right)$. It's easy to see that if $r_{m_{b}}$ is selected uniformly randomly from $Z_{q^{\prime}}$, then $g^{r_{m_{b}}+\sigma_{m_{b}}} h^{r_{m_{b}}}$ is distributed uniformly in $G_{q^{\prime}}$. We will show that if $\operatorname{Succ}_{\mathcal{A}, \mathcal{K} \mathcal{E}}$ is non-negligible, then there is an attacker $\mathcal{B}$ who can win the following game with a non-negligible probability $\operatorname{Succ}_{\mathcal{B}, \mathcal{C}}$, which contradicts the fact that $r$ is random.

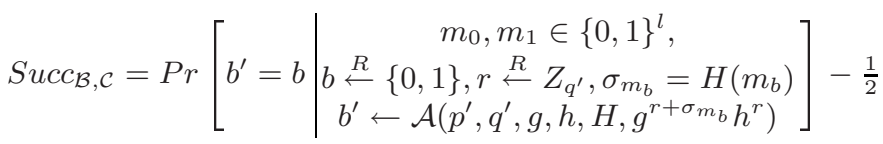

$\mathcal{B}$ first sends $m_{0}, m_{1}$ to the encryption oracle and receives $g^{r+\sigma_{m_{b}}} h^{r}$. Then it chooses a random number $a \in Z_{q^{\prime}}$ and generates $n$ pairs of $\left(a_{i 1}, a_{i 2}\right)$ such that $a_{i 1} a_{i 2} \equiv a \bmod p^{\prime}$. It also computes $\sigma_{m_{0}}=H\left(m_{0}\right)$ and $\theta=g^{r+\sigma_{m_{b}}} h^{r} g^{-\sigma_{m_{0}}}$, it is clear that $\operatorname{Pr}\left[\theta=g^{r} h^{r}\right]=\frac{1}{2}$. Then $\mathcal{B}$ sends $\left(m_{0}, m_{1}, p^{\prime}, q^{\prime}, g, h, g^{a} h^{a}\right.$, $\left.\left(g^{r+\sigma_{m_{b}}} h^{r}\right)^{a_{11}},\left(g^{r+\sigma_{m_{b}}} h^{r}\right)^{a}, H\left(\theta^{a}\right), a_{12}, \ldots, a_{n 2}\right)$ to $\mathcal{A}$. If $\theta=g^{r} h^{r}$, then $\mathcal{A}$ can

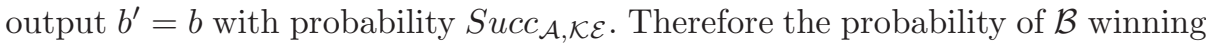

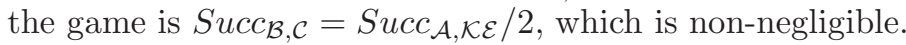

The semantically secure definition for searchable encryption is tricky because searching leaks information inevitably. As long as the searching algorithm is correct, it always returns the same result set for the same query. Although the queries and the result sets are encrypted, the adversary can still build up search patterns. Therefore the security definition for searchable encryption should be modified to reflect the intuition that nothing should be leaked beyond the outcome and the pattern of a sequence of searches. Here we adapt the definition from [11] and prove our scheme is non-adaptive semantically secure. Informally, non-adaptive semantic security means that given two non-adaptively generated query histories with the same length and outcome, no PPT adversary can distinguish one from another with non-negligible probability. Non-adaptive means the adversary cannot choose queries based on the prior queries and results. This is acceptable because in our setting, only the authorised user can generate queries.

We first introduce some notions to be used in the definition. $\Delta$ is the set of all possible data items, i.e. documents. $\mathcal{D}=\left\{D_{1}, \ldots, D_{n}\right\}$ denotes an arbitrary 
subset of $\Delta$, i.e. $\mathcal{D} \in \mathcal{P}(\Delta)$, and each $D_{i}$ is a document. $\mathcal{W}=\left\{w_{1}, \ldots, w_{d}\right\}$ is a dictionary which contains all the possible words can be used in the queries. Each document in $\mathcal{D}$ is associated with a local unique identifier $i d\left(D_{i}\right)$, and a set of keywords $k w\left(D_{i}\right)$ which is a subset of $\mathcal{W}$. The result set of a search query $w$ on a document set is denoted by $r s(w)$, which is the set of document identifiers of all the documents in $\mathcal{D}$ that contain the keyword, i.e. $\{i d(D) \mid D \in \mathcal{D} \wedge w \in k w(D)\}$. A history is defined in terms of a sequence of queries made on a document set.

Definition 2 (History). A history $H_{q} \in \mathcal{P}(\Delta) \times \mathcal{W}^{q}$ is an interaction between a client and a server over $q$ queries on a document set $\mathcal{D}$, i.e. $H_{q}=\left(\mathcal{D}, w_{1}, \ldots, w_{q}\right)$.

During the interaction, the adversary cannot directly see the history because the documents, keywords and queries are encrypted. What the adversary can see is a view, i.e. the encrypted version of the history. Let $E$ be the symmetric key encryption scheme, $\mathcal{E}$ be the proxy encryption scheme and $\mathcal{K} \mathcal{E}$ be the keyword encryption scheme, $Q_{i}$ be an encrypted query, the view of the adversary is then defined as:

Definition 3 (View). Given a document set $\mathcal{D}$ with $n$ documents and a history over $q$ queries $H_{q}=\left(\mathcal{D}, w_{1}, \ldots, w_{q}\right)$, an adversary's view of $H_{q}$ is defined as: $V\left(H_{q}\right)=\left(i d\left(D_{1}\right), \ldots, i d\left(D_{n}\right), E_{k_{1}}\left(D_{1}\right), \ldots, E_{k_{n}}\left(D_{n}\right), \mathcal{E}\left(k_{1}\right), \ldots, \mathcal{E}\left(k_{n}\right), \mathcal{K} \mathcal{E}\left(k w\left(D_{1}\right)\right), \ldots\right.$, $\left.\mathcal{K} \mathcal{E}\left(k w\left(D_{n}\right)\right), Q_{1}, \ldots, Q_{q}\right)$.

As we have stated above, searching leaks information. The maximum information we have to leak is captured by trace. In our settings, a trace contains information from three sources: the encrypted file stored on the server, e.g. the id, length and number of keywords of each document, the result set and the query pattern.

Definition 4 (Trace). Given a document set $\mathcal{D}$ with $n$ documents and a history over $q$ queries $H_{q}$, the trace of $H_{q}$ is defined as:

$\operatorname{Tr}\left(H_{q}\right)=\left(i d\left(D_{1}\right), \ldots, i d\left(D_{n}\right),\left|D_{1}\right|, \ldots,\left|D_{n}\right|,\left|k w\left(D_{1}\right)\right|, \ldots,\left|k w\left(D_{n}\right)\right|, r s\left(w_{1}\right), \ldots, r s\left(w_{q}\right), \Pi_{q}\right)$. $\Pi_{q}$ is the search pattern over the history which is a symmetric binary matrix where $\Pi_{q}[i, j]=1$ if $w_{i}=w_{j}$, and $\Pi_{q}[i, j]=0$ otherwise, for $1 \leq i, j \leq q$.

The security definition is then based on the idea that the scheme is secure if no more information is leaked beyond what the adversary can get from the traces. This intuition is formalised by defining a game where the adversary has to distinguish two histories, possibly on two different document sets, which have the same trace. Since the traces are identical, the adversary cannot distinguish the two histories by the traces, i.e. the knowledge he already has. He must extract additional knowledge from what he can see during the interactions, i.e. the views. The negligible probability of the adversary successfully distinguishing the two histories implies that he cannot get extra knowledge and in consequence the scheme is secure.

Definition 5 (Non-Adaptive Semantic Security). Our searchable data encryption is Non-Adaptive Semantically Secure if for all $q \in \mathbb{N}$, for all $\left(H_{0}, H_{1}\right)$ which are histories over $q$ queries and $\operatorname{Tr}\left(H_{0}\right)=\operatorname{Tr}\left(H_{1}\right)$, and any PPT adver$\operatorname{sary}_{\mathcal{A}}, \operatorname{Succ}_{\mathcal{A}}$ is negligible: 


$$
\operatorname{Succ}_{\mathcal{A}}=\operatorname{Pr}\left[b^{\prime}=b \mid \begin{array}{c}
\text { Pub_para, Sec_para }, \mathcal{K}_{u}, \mathcal{K}_{p} \leftarrow \operatorname{SETUP}\left(1^{k}\right), \\
H_{0}, H_{1} \in \mathcal{P}(\Delta) \times \mathcal{W}^{q}, \\
b \stackrel{R}{\leftarrow}\{0,1\}, \\
b^{\prime} \leftarrow \mathcal{A}\left(\text { Pub_para }, \mathcal{K}_{p}, V\left(H_{b}\right)\right)
\end{array}\right]-\frac{1}{2}
$$

Theorem 2. The enhanced construction is non-adaptive semantically secure.

Proof. Let's examine each part of the view.

Document identifiers $i d\left(D_{1}\right), \ldots, i d\left(D_{n}\right)$ : Because $\operatorname{Tr}\left(H_{0}\right)=\operatorname{Tr}\left(H_{1}\right)$, this part of the view must be identical for the two histories. So the adversary cannot distinguish the two histories by the document identifiers.

Encrypted documents $E_{k_{1}}\left(D_{1}\right), \ldots, E_{k_{n}}\left(D_{n}\right)$ : The adversary cannot distinguish because $E$ is semantically secure.

Encrypted symmetric keys $\mathcal{E}\left(k_{1}\right), \ldots, \mathcal{E}\left(k_{n}\right): \mathcal{E}$ is based on RSA-OAEP which is IND-CCA2 secure. Therefore is also indistinguishable.

Encrypted keywords $\mathcal{K} \mathcal{E}\left(k w\left(D_{1}\right)\right), \ldots, \mathcal{K} \mathcal{E}\left(k w\left(D_{n}\right)\right)$ : We have proved they are indistinguishable to the adversary in lemma 2 .

Encrypted queries $Q_{1}, \ldots, Q_{q}$ : Because $\operatorname{Tr}\left(H_{0}\right)=\operatorname{Tr}\left(H_{1}\right)$, we don't need to consider the query pattern and can reduce the problem to distinguish any two sequences of distinct queries: $\left(Q_{01}, \ldots, Q_{0 m}\right),\left(Q_{11}, \ldots, Q_{1 m}\right), m \leq q$. For each $Q_{i j}, i \in$ $0,1,1 \leq j \leq m$, it is a pseudorandom number $g^{a_{1} H\left(w_{i j}\right)} \bmod p^{\prime}$. Therefore the queries are not distinguishable as long as the discrete logarithm problem is hard.

\section{Other Considerations}

Access to encrypted data involves both client-side and server-side keys. So revoking a user's access is quite simple. The KMS can send an instruction to the server to let it remove the user's corresponding keys on the server side. After the keys have been removed, the user cannot access the data unless the KMS generates new keys for him. Even a revoked user can masquerade as an authorised user, his requests cannot be processed correctly if he does not know the authorised user's keys.

Each authorised user has his own RSA key pair $\left(e_{i 1}, d_{i 1}\right)$ and the server holds the corresponding key pair $\left(e_{i 2}, d_{i 2}\right)$. Because $e_{i 1} d_{i 1} e_{i 2} d_{i 2} \equiv e d \equiv 1 \bmod \phi(n)$, $k_{1}=e_{i 1} d_{i 1}$ and $k_{2}=e_{i 2} d_{i 2}$ form another RSA key pair. This key pair can be used for public key based mutual authentication and to establish a secure channel e.g. SSL. This adds another layer of protection against unauthorised users.

The main concern with proxy encryption schemes comes from a collusion attack. If a user colludes with adversary $A d v$, who knows all the server side keys, they can easily recover the master keys by combining their keys. Although some work has been done in [16] using bilinear map to prevent the colluded parties from recovering the master key, the colluded parties are still able to decrypt the ciphertext with a weak secret they can recover. Theoretically, the design of collusion-resistant proxy encryption schemes is an open problem. But in practice, we can lower the risk to an acceptable level by implementing other mechanisms. For example, we can limit the access to the keys by using tamper-proof devices. 


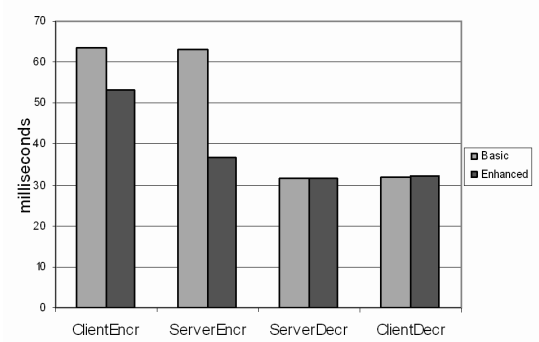

(a) Client and Server Encryption/ Decryption Operations

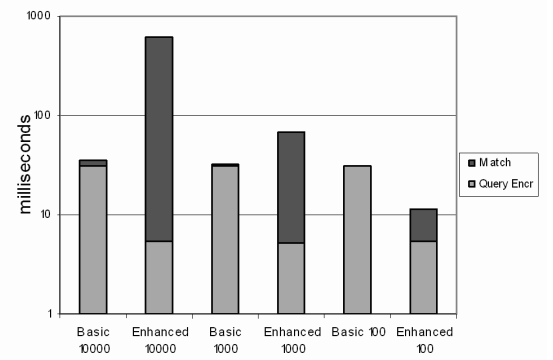

(b) Search Operation for different Repository Sizes

Fig. 4. Performance of the Operations

We can also split the master keys into multiple shares and introduce additional servers, making collusion more difficult. Monitoring and auditing to detect collusion can also help to mitigate the risk.

\section{Implementation and Performance}

We implemented a prototype in Java using the packages provided in the standard Java 1.5 distribution. We use our encryption scheme to encrypt a single table database. We chose AES as the symmetric cipher which encrypts the actual data and SHA-1 as the hash function. For the RSA-based proxy encryption scheme, we used 1024-bit keys. For the keyword encryption scheme, $q^{\prime}$ was 160 -bit and $p^{\prime}$ was 1024-bit. The tests were executed on a Intel Pentium IV $3.2 \mathrm{GHz}$ (dual core) with $1 \mathrm{~GB}$ of RAM.

The first evaluation consists of measuring for each scheme the execution time of the following operations: (1) Client Encryption: that consists of encrypting a data item using the symmetric cipher, encrypting the symmetric key and encrypting the keywords; (2) Server Encryption: re-encryption of the symmetric key and the keywords using the server side keys; (3) Server Decryption: predecryption of the symmetric key; (4) Client Decryption: decryption of the symmetric key and the data item.

The graph in Fig. 4 (a) shows the performance for the execution of encryption and decryption operations for each construction. The time in the Y-axis is given in milliseconds. The graph provides the average time for 10,000 executions. The data item we used in the experiments was a 16-byte string with one associated keyword. The result shows that the enhanced construction has better performance than the basic construction in encryption. Since the data encryption and key encryption are nearly identical in both constructions, the difference is due to the fact that they encrypt the keywords using different schemes. The enhanced construction encrypts the keywords using a Discrete Logarithm based scheme and the basic construction uses an RSA-based scheme. The exponent used in the DL scheme is smaller than that of the RSA scheme, therefore the keyword encryption of the DL scheme is faster than the RSA scheme. The decryption 
part of both constructions are almost the same, so we can see from the figure that the two constructions have nearly the same performance in decryption.

We also measured the time for processing a search query on the server side in both constructions, the result is shown in Fig. 44(b). Processing a search query involves two operations: query re-encryption (Query Encr) and matching (Match). The graph shows the time (in milliseconds) for a search operation (the time scale is logarithmic) executed on several databases with different sizes in both constructions. We used three databases containing 100, 1,000 and 10,000 keywords each. The graph shows that for the basic construction, the query encryption dominates the overall searching time. This is easy to understand since the matching operation in the basic construction is simply string comparison. Therefore the size of the database has little effect on the searching time in the basic construction. In contrast, the time spent on the matching operation is much more significant in the enhanced construction. And when the database becomes large, the time increases linearly. As a result, the basic construction has better performance than the enhanced construction when searching large databases.

\section{Conclusion and Future Work}

In this paper, we presented a new data encryption scheme that does not require a trusted data server. In the scheme the server can perform searches and updates on the encrypted data without knowing the plaintext or the decryption keys. Unlike previous searchable data encryption schemes that require a shared key for multi-user access, each user in our system has a unique set of keys. The data encrypted by one user can be correctly decrypted by all the authorised users in the system. Moreover the keys can be easily revoked without any overhead, i.e. without having to re-encrypt the stored data. We provided two constructions for the scheme built on top of proxy encryption schemes. For each construction, we gave the formal definitions and proofs of security. We also implemented them in Java and compared the performance.

One aspect of our future work is to investigate and integrate our scheme with Private Information Retrieval (PIR) schemes. PIR schemes [24 25 26] allow a user to retrieve some items from a database without revealing to the database which items were queried. A weakness of our scheme is that it allows statistical attacks on the queries. By combining PIR techniques, we could potentially make our scheme more secure. Secure indexes 45] is another promising technique that is used to improve the performance and decrease the storage overhead of searchable encryption schemes. We will investigate current schemes and develop a new index scheme for the multi-user system.

\section{References}

1. Blackwood, J.: Is storage outsourcing a viable alternative? http://techupdate. zdnet.com/techupdate/stories/main/0,14179,2851289,00.html

2. Connor, D.: Storage outsourcing on the rise, http://www.networkworld.com/ news/2007/012207-storage-outsourcing-rises.html 
3. Song, D.X., Wagner, D., Perrig, A.: Practical techniques for searches on encrypted data. In: IEEE Symposium on Security and Privacy, pp. 44-55 (2000)

4. Yang, Z., Zhong, S., Wright, R.N.: Privacy-Preserving Queries on Encrypted Data. In: Gollmann, D., Meier, J., Sabelfeld, A. (eds.) ESORICS 2006. LNCS, vol. 4189, pp. 479-495. Springer, Heidelberg (2006)

5. Goh, E.J.: Secure indexes. Cryptology ePrint Archive, Report 2003/216 (2003), http://eprint.iacr.org/2003/216/

6. Hacigümüs, H., Iyer, B.R., Li, C., Mehrotra, S.: Executing sql over encrypted data in the database-service-provider model. In: SIGMOD Conference, pp. 216227 (2002)

7. Damiani, E., di Vimercati, S.D.C., Jajodia, S., Paraboschi, S., Samarati, P.: Balancing confidentiality and efficiency in untrusted relational dbmss. In: ACM Conference on Computer and Communications Security, pp. 93-102 (2003)

8. Agrawal, R., Kiernan, J., Srikant, R., Xu, Y.: Order-preserving encryption for numeric data. In: SIGMOD Conference, pp. 563-574 (2004)

9. Hore, B., Mehrotra, S., Tsudik, G.: A privacy-preserving index for range queries. In: VLDB, pp. 720-731 (2004)

10. Boneh, D., Crescenzo, G.D., Ostrovsky, R., Persiano, G.: Public Key Encryption with Keyword Search. In: Cachin, C., Camenisch, J.L. (eds.) EUROCRYPT 2004. LNCS, vol. 3027, pp. 506-522. Springer, Heidelberg (2004)

11. Curtmola, R., Garay, J.A., Kamara, S., Ostrovsky, R.: Searchable symmetric encryption: improved definitions and efficient constructions. In: ACM Conference on Computer and Communications Security, pp. 79-88 (2006)

12. Blaze, M., Bleumer, G., Strauss, M.: Divertible Protocols and Atomic Proxy Cryptography. In: Nyberg, K. (ed.) EUROCRYPT 1998. LNCS, vol. 1403, pp. 127-144. Springer, Heidelberg (1998)

13. Elgamal, T.: A public key cryptosystem and a signature scheme based on discrete logarithms. IEEE Transactions on Information Theory 31(4), 469-472 (1985)

14. Rivest, R.L., Shamir, A., Adleman, L.M.: A method for obtaining digital signatures and public-key cryptosystems. Commun. ACM 21(2), 120-126 (1978)

15. Khurana, H., Slagell, A.J., Bonilla, R.: Sels: a secure e-mail list service. In: SAC, pp. 306-313 (2005)

16. Ateniese, G., Fu, K., Green, M., Hohenberger, S.: Improved proxy re-encryption schemes with applications to secure distributed storage. In: NDSS (2005)

17. Kapadia, A., Tsang, P.P., Smith, S.W.: Attribute-based publishing with hidden credentials and hidden policies. In: NDSS (2007)

18. Ivan, A.A., Dodis, Y.: Proxy cryptography revisited. In: NDSS (2003)

19. Goldreich, O.: Foundations of Cryptography: Basic Applications, vol. II. Cambridge University Press, Cambridge (2004)

20. Simmons, G.J.: A "weak" privacy protocol using the rsa crypto algorithm. Cryptologia 7(2), 180-182 (1983)

21. Delaurentis, J.M.: A further weakness in the common modulus protocol for the rsa cryptoalgorithm. Cryptologia 8(3), 253-259 (1984)

22. Bellare, M., Rogaway, P.: Optimal Asymmetric Encryption. In: De Santis, A. (ed.) EUROCRYPT 1994. LNCS, vol. 950, pp. 92-111. Springer, Heidelberg (1995)

23. Fujisaki, E., Okamoto, T., Pointcheval, D., Stern, J.: RSA-OAEP Is Secure under the RSA Assumption. In: Kilian, J. (ed.) CRYPTO 2001. LNCS, vol. 2139, pp. 260-274. Springer, Heidelberg (2001) 
24. Chor, B., Goldreich, O., Kushilevitz, E., Sudan, M.: Private information retrieval. In: FOCS, pp. 41-50 (1995)

25. Cachin, C., Micali, S., Stadler, M.: Computationally Private Information Retrieval with Polylogarithmic Communication. In: Stern, J. (ed.) EUROCRYPT 1999. LNCS, vol. 1592, pp. 402-414. Springer, Heidelberg (1999)

26. Gentry, C., Ramzan, Z.: Single-Database Private Information Retrieval with Constant Communication Rate. In: Caires, L., Italiano, G.F., Monteiro, L., Palamidessi, C., Yung, M. (eds.) ICALP 2005. LNCS, vol. 3580, pp. 803-815. Springer, Heidelberg (2005) 